PROCEEDINGS OF THE

AMERICAN MATHEMATICAL SOCIETY

Volume 137, Number 9, September 2009, Pages 2979-2987

S 0002-9939(09)09932-8

Article electronically published on April 14, 2009

\title{
ORTHOGONAL POLYNOMIALS AND PARTIAL DIFFERENTIAL EQUATIONS ON THE UNIT BALL
}

\author{
MIGUEL PIÑAR AND YUAN XU \\ (Communicated by Peter A. Clarkson)
}

\begin{abstract}
Orthogonal polynomials of degree $n$ with respect to the weight function $W_{\mu}(x)=\left(1-\|x\|^{2}\right)^{\mu}$ on the unit ball in $\mathbb{R}$ are known to satisfy the partial differential equation

$$
\left[\Delta-\langle x, \nabla\rangle^{2}-(2 \mu+d)\langle x, \nabla\rangle\right] P=-n(n+2 \mu+d) P
$$

for $\mu>-1$. The singular case of $\mu=-1,-2, \ldots$ is studied in this paper. Explicit polynomial solutions are constructed and the equation for $\nu=-2,-3, \ldots$ is shown to have complete polynomial solutions if the dimension $d$ is odd. The orthogonality of the solution is also discussed.
\end{abstract}

\section{INTRODUCTION}

Let $W_{\mu}(x)=\left(1-\|x\|^{2}\right)^{\mu}$. For $\mu>-1, W_{\mu}$ is integrable on the unit ball $B^{d}:=\{x:\|x\| \leq 1\}$ and orthogonal polynomials with respect to $W_{\mu}$ are well defined. We denote by $\mathcal{V}_{n}^{d}\left(W_{\mu}\right)$ the space of orthogonal polynomials of degree $n$ with respect to $W_{\mu}$ on $B^{d}$. It is well known (cf. [5]) that $P \in \mathcal{V}_{n}^{d}\left(W_{\mu}\right)$ satisfies the partial differential equation

$$
\begin{aligned}
L_{\mu} P & :=\left[\Delta-\langle x, \nabla\rangle^{2}-(2 \mu+d)\langle x, \nabla\rangle\right] P=\lambda_{n}^{(\mu)} P, \quad \text { with } \\
\lambda_{n}^{(\mu)} & :=-n(n+2 \mu+d),
\end{aligned}
$$

where $\Delta$ denotes the usual Laplacian, $\nabla=\left(\partial_{1}, \ldots, \partial_{d}\right)$ with $\partial_{i}=\frac{\partial}{\partial x_{i}}$ so that $\langle x, \nabla\rangle=\sum_{i=1}^{d} x_{i} \frac{\partial}{\partial x_{i}}$. In other words, the elements of $\mathcal{V}_{n}^{d}\left(W_{\mu}\right)$ are eigenfunctions of $L_{\mu}$ with eigenvalues $\lambda_{n}^{(\mu)}$. Since $\bigcup_{n \geq 0} \mathcal{V}_{n}^{d}\left(W_{\mu}\right)$ contains a basis for all polynomials, we say that $L_{\mu}$ has complete polynomial solutions for $\mu>-1$.

The purpose of this paper is to study equation (1.1) for $\mu=-1,-2, \ldots$ Our study starts from the realization that orthogonal polynomials with respect to the

Received by the editors December 18, 2007.

2000 Mathematics Subject Classification. Primary 33C50, 33E30, 42C05.

Key words and phrases. pde, orthogonal polynomials, several variables, unit ball.

Partially supported by Ministerio de Ciencia y Tecnología (MCYT) of Spain and by the European Regional Development Fund (ERDF) through the grant MTM 2005-08648-C02-02, and Junta de Andalucía, Grupo de Investigación FQM 0229. The work of the second author was supported in part by NSF Grant DMS-0604056.

(C)2009 American Mathematical Society Reverts to public domain 28 years from publication 
inner product

$$
\langle f, g\rangle_{-1}=\lambda \int_{B^{d}} \nabla f(x) \cdot \nabla g(x) d x+\int_{S^{d-1}} f(x) g(x) d \omega, \quad \lambda>0,
$$

which were studied recently in [12], satisfy (1.1) for $\mu=-1$ (see Section 2). To verify this, explicit orthogonal structure from [12] is used. It turns out that it is possible to construct explicit polynomial solutions for the equation (1.1) when $-\mu \in \mathbb{N}$. However, somewhat surprisingly, (1.1) has a complete set of polynomial solutions only when the dimension $d$ is odd, and our construction shows why this is so. A natural question is whether the polynomial solutions of (1.1) remain orthogonal for $\mu=-2,-3, \ldots$ when $d$ is odd. We do not know the answer to this question. We are, however, able to show that a family of polynomials closely related to the solutions of the equation (1.1) for $k=-2$ are orthogonal with respect to

$$
\langle f, g\rangle_{-2}=\lambda \int_{B^{d}} \Delta f(x) \Delta g(x) d x+\int_{S^{d-1}} f(x) g(x) d \omega, \quad \lambda>0 .
$$

Furthermore, for each $n \in \mathbb{N}$, the polynomial solutions of (1.1) for $k=-2$ are orthogonal with respect to an inner product that is a modification of $\langle\cdot, \cdot\rangle_{-2}$ but depends on $n$.

Historically, only the case $d=1$ of such a problem has been thoroughly studied. In the case of $d=1$, the equation (1.1) becomes the ordinary differential equation satisfied by the Gegenbauer polynomials. In this case, the problem of negative indices has been studied by several authors; we refer to [1, 2, 3, 6] and the references therein. For $d=2$, equation (1.1) is classical and can be traced back to Hermite. It is one of five second-order partial differential equations with polynomial coefficients that have orthogonal polynomial solutions with respect to a positive definite moment functional, as classified by Krall and Scheffer in [7]. We note that our result for $\mu=-1$ does not conflict with the result of [7], since $\langle f, g\rangle_{-1}$ does not come from a moment functional. For $d=2$, the case $-\mu \notin \mathbb{N}$ is considered in [9], where the fact that (1.1) does not have complete polynomial solutions for $-\mu \in \mathbb{N}$ is also observed. Finally, we should mention [8] and 11] for relevant results.

The paper is organized as follows. We collect background materials in the following section. The solutions of (1.1) for $\mu \in \mathbb{N}$ are constructed in Section 3 . Orthogonal polynomials with respect to $\langle\cdot, \cdot\rangle_{-2}$ are discussed in Section 4.

\section{BACKGROUND AND PRELIMINARY}

Let $d \omega$ denote the Lebesgue measure on $S^{d-1}:=\{x:\|x\|=1\}$ and denote the area of $S^{d-1}$ by $\omega_{d}, \omega_{d}:=\int_{S^{d-1}} d \omega=2 \pi^{d / 2} / \Gamma(d / 2)$. We start with the spherical harmonics, which are homogeneous polynomials satisfying the equation $\Delta P=0$. Let $\mathcal{H}_{n}^{d}$ denote the space of homogeneous harmonic polynomials of degree $n$. It is well known that

$$
\operatorname{dim} \mathcal{H}_{n}^{d}=\left(\begin{array}{c}
n+d-1 \\
d-1
\end{array}\right)-\left(\begin{array}{c}
n+d-3 \\
d-1
\end{array}\right):=\sigma_{n}
$$

The restriction of $Y \in \mathcal{H}_{n}^{d}$ on $S^{d-1}$ gives the spherical harmonics, which are orthogonal on $S^{d-1}$. Throughout this paper, we use the notation $\left\{Y_{\nu}^{n}: 1 \leq \nu \leq \sigma_{n}\right\}$ to denote an orthonormal basis for $\mathcal{H}_{n}^{d}$, that is,

$$
\frac{1}{\omega_{d}} \int_{S^{d-1}} Y_{\mu}^{n}\left(x^{\prime}\right) Y_{\nu}^{m}\left(x^{\prime}\right) d \omega\left(x^{\prime}\right)=\delta_{\mu, \nu} \delta_{n, m}, \quad 1 \leq \mu, \nu \leq \sigma_{n} .
$$


We will also denote by $\Pi_{n}^{d}$ the space of polynomials of degree $n$ in $d$ variables and by $\mathcal{P}_{n}^{d}$ the space of homogeneous polynomials of degree $n$. It is well known that $\operatorname{dim} \mathcal{P}_{n}^{d}=\left(\begin{array}{c}n+d-1 \\ d-1\end{array}\right)$.

For orthogonal polynomials on the unit ball, we start with the inner product

$$
\langle f, g\rangle_{\mu}=c_{\mu} \int_{B^{d}} f(x) g(x)\left(1-\|x\|^{2}\right)^{\mu} d x, \quad \mu>-1,
$$

where $c_{\mu}$ is the normalization constant of $W_{\mu}$. Let $\mathcal{V}_{n}^{d}\left(W_{\mu}\right)$ denote the space of orthogonal polynomials of degree $n$. A mutually orthogonal basis for $\mathcal{V}_{n}^{d}\left(W_{\mu}\right)$ is given by $([5])$

$$
P_{j, \nu}^{n}\left(W_{\mu} ; x\right)=P_{j}^{\left(\mu, n-2 j+\frac{d-2}{2}\right)}\left(2\|x\|^{2}-1\right) Y_{\nu}^{n-2 j}(x), \quad 0 \leq j \leq n / 2,
$$

where $P_{j}^{(\alpha, \beta)}$ denotes the Jacobi polynomial of degree $j$, which is orthogonal with respect to $(1-x)^{\alpha}(1+x)^{\beta}$ on $[-1,1]$, and $\left\{Y_{\nu}^{n-2 j}: 1 \leq \nu \leq \sigma_{n-2 j}\right\}$ is an orthonormal basis for $\mathcal{H}_{n-2 j}^{d}$.

We will need orthogonal polynomials for two other inner products on the ball. One is $\langle\cdot, \cdot\rangle_{-1}$ in the introduction, which we normalize as

$$
\langle f, g\rangle_{-1}:=\frac{\lambda}{\omega_{d}} \int_{B^{d}} \nabla f(x) \cdot \nabla g(x) d x+\frac{1}{\omega_{d}} \int_{S^{d-1}} f(x) g(x) d \omega_{d}, \quad \lambda>0,
$$

so that $\langle 1,1\rangle_{-1}=1$. Let $\mathcal{V}_{n}^{d}\left(W_{-1}\right)$ denote the space of orthogonal polynomials with respect to $\langle 1,1\rangle_{-1}$. Then, as shown in [12],

$$
\mathcal{V}_{n}^{d}\left(W_{-1}\right)=\mathcal{H}_{n}^{d} \oplus\left(1-\|x\|^{2}\right) \mathcal{V}_{n-2}^{d}\left(W_{1}\right) .
$$

The second inner product is motivated by an application in the numerical solution of Poisson equations (see [4]) and it is defined by

$$
\langle f, g\rangle_{\Delta}=\alpha_{d} \int_{B^{d}} \Delta\left[\left(1-\|x\|^{2}\right) f(x)\right] \Delta\left[\left(1-\|x\|^{2}\right) g(x)\right] d x,
$$

where $a_{d}=1 /\left(4 d^{2} \operatorname{vol}\left(B^{d}\right)\right)$ so that $\langle 1,1\rangle_{\Delta}=1$. Let $\mathcal{V}_{n}^{d}(\Delta)$ denote the space of orthogonal polynomials with respect to $\langle\cdot, \cdot\rangle_{\Delta}$. Then, as shown in [11,

$$
\mathcal{V}_{n}^{d}(\Delta)=\mathcal{H}_{n}^{d} \oplus\left(1-\|x\|^{2}\right) \mathcal{V}_{n-2}^{d}\left(W_{2}\right)
$$

We refer to 11, 12 for further properties of orthogonal polynomials with respect to these two inner products. Note that an explicit orthonormal basis for either orthogonal polynomial subspace can be easily deduced from (2.2).

In the above and throughout this paper, we write $g(x) V_{n}=\{g(x) p(x): p \in$ $\left.V_{n}\right\}$, where $V_{n}$ is either the subspace $\mathcal{H}_{n}^{d}$ of harmonic polynomials or the subspace $\mathcal{V}_{n}^{d}\left(W_{\mu}\right)$ of orthogonal polynomials. Furthermore, if $n<0$, then we define $\mathcal{H}_{n}^{d}=\emptyset$ and $\mathcal{V}_{n}^{d}=\emptyset$.

\section{Polynomial solutions of the partial differential equation}

We start with two lemmas on the operator $L_{\mu} f$.

Lemma 3.1. For $\mu \in \mathbb{R}$,

$$
L_{\mu}\left[\left(1-\|x\|^{2}\right) P\right]=4(\mu+1) P+\left(1-\|x\|^{2}\right)\left[-(4(\mu+1)+2 d) P+L_{\mu+2} P\right] .
$$


Proof. The proof follows from a straightforward computation. The essential ingredients are

$$
\begin{aligned}
\Delta\left[\left(1-\|x\|^{2}\right) P\right]= & -2 d P-4\langle x, \nabla\rangle P+\left(1-\|\left. x\right|^{2}\right) P, \\
\langle x, \nabla\rangle\left[\left(1-\|x\|^{2}\right) P\right]= & -2 P+2\left(1-\|x\|^{2}\right) P+\left(1-\|x\|^{2}\right)\langle x, \nabla\rangle P+\left(1-\|\left. x\right|^{2}\right) P, \\
\langle x, \nabla\rangle^{2}\left[\left(1-\|x\|^{2}\right) P\right]= & -4 P-4\langle x, \nabla\rangle P+4\left(1-\|x\|^{2}\right) P \\
& +4\left(1-\|x\|^{2}\right)\langle x, \nabla\rangle P+\left(1-\|\left. x\right|^{2}\right)\langle x, \nabla\rangle^{2} P,
\end{aligned}
$$

as can be easily verified.

Lemma 3.2. For all $\mu \in \mathbb{R}$ and $n \geq 0, L_{\mu} Y=\lambda_{n}^{(\mu)} Y$ for $Y \in \mathcal{H}_{n}^{d}$.

Proof. If $Y \in \mathcal{H}_{n}^{d}$, then $\Delta Y=0$. Since $Y$ is homogeneous, Euler's formula shows that $\langle x, \nabla\rangle Y=n Y$. The stated result follows immediately from these two facts.

Our first result shows that orthogonal polynomials with respect to $\langle\cdot, \cdot\rangle_{-1}$ satisfy the equation (1.1) with $\mu=-1$.

Theorem 3.3. Elements of $\mathcal{V}_{n}^{d}\left(W_{-1}\right)$ satisfy $L_{-1} P=\lambda_{n}^{(-1)} P$. In particular, the eigenfunctions of the operator $L_{-1}$ consist of a complete polynomial basis.

Proof. Setting $\mu=-1$, equation (3.1) shows that

$$
L_{-1}\left[\left(1-\|x\|^{2}\right) P\right]=\left(1-\|x\|^{2}\right)\left[-2 d P+L_{1} P\right] .
$$

Recall that elements of $\mathcal{V}_{n-2}^{d}\left(W_{1}\right)$ satisfy the equation (1.1) with $\mu=1$. It follows that for $P \in \mathcal{V}_{n-2}^{d}\left(W_{1}\right)$,

$$
\begin{aligned}
L_{-1}\left[\left(1-\|x\|^{2}\right) P\right] & =\left(1-\|x\|^{2}\right)[-2 d P-(n-2)(n+2 \mu+d-2) P] \\
& =-n(n+d-2)\left(1-\|x\|^{2}\right) P .
\end{aligned}
$$

By (2.3), this and Lemma 3.2 show that all elements in $\mathcal{V}_{n}^{d}\left(W_{-1}\right)$ satisfy (1.1) for $\mu=-1$.

For $d=2$, a complete characterization of second-order linear partial differential equations that have polynomial coefficients and orthogonal polynomials as eigenfunctions is given in [7, where the orthogonality is given in terms of a moment functional. If the moment functional is positive definite, then there are only five families of equations; the equation (1.1) is one of them when $\mu>-1$. It should be remarked that Theorem 3.3 does not contradict the characterization of [7, since the inner product $\langle\cdot, \cdot\rangle_{-1}$ cannot be expressed as a moment functional. Indeed, it is easy to see that $\langle x f, g\rangle_{-1} \neq\langle f, x g\rangle_{-1}$ in general.

The above theorem prompts us to consider solutions of (1.1) for $\mu=-2,-3, \ldots$ and leads to our second theorem. For $\mu=-k, k=2,3, \ldots$, we define

$$
\mathcal{U}_{n}^{d}\left(W_{-k}\right):=\mathcal{H}_{n}^{d} \cup\left(\bigcup_{j=1}^{k-1}\left[\sum_{\nu=0}^{j} a_{j, \nu}^{n}\left(1-\|x\|^{2}\right)^{\nu}\right] \mathcal{H}_{n-2 j}^{d}\right) \cup\left(1-\|x\|^{2}\right)^{k} \mathcal{V}_{n-2 k}^{d}\left(W_{k}\right),
$$

where, for $1 \leq j \leq k-1$,

$$
a_{j, \nu}^{n}:=\frac{(-1)^{j-\nu} j !(-k+1)_{j}\left(n-j-k+\frac{d}{2}\right)_{\nu}}{\nu !(j-\nu) !(-k+1)_{\nu}\left(n-j-k+\frac{d}{2}\right)_{j}}, \quad 0 \leq \nu \leq j,
$$

in which $(a)_{m}:=a(a+1) \ldots(a+m-1)$ is the shifted factorial. We note that $a_{j, j}^{n}=1$. It is easy to see that $a_{j, \nu}^{n}$ are well defined if $n-j-k+\nu+\frac{d}{2}$ is not zero. 
Theorem 3.4. If $\mu=-k$ and $k=2,3, \ldots$, then the polynomials in $\mathcal{U}_{n}^{d}\left(W_{-k}\right)$ satisfy equation (1.1); that is, $L_{-k} P=\lambda_{n}^{(-k)} P$ for $P \in \mathcal{U}_{n}^{d}\left(W_{-k}\right)$. Furthermore,

$$
\operatorname{dim} \mathcal{U}_{n}^{d}=\operatorname{dim} \mathcal{P}_{n}^{d}, \quad \text { if } n-2 k-1+\frac{d}{2} \neq 0 .
$$

In particular, the operator $L_{-k}$ has a complete polynomial basis of eigenfunctions if the dimension $d$ is odd.

Proof. We observe that the relation (3.1) can be used recursively, which yields

$$
\begin{aligned}
L_{\mu}\left(1-\|x\|^{2}\right)^{k}= & 4 k(\mu+k)\left(1-\|x\|^{2}\right)^{k-1} \\
& +\left(1-\|x\|^{2}\right)^{k}\left[-(4 k(\mu+k)+2 k d)+L_{\mu+2 k}\right], \quad k \in \mathbb{N},
\end{aligned}
$$

as can be easily verified by induction. Hence, for $Q \in \mathcal{V}_{n-2 k}^{d}\left(W_{k}\right)$, which satisfies $L_{k} Q=\lambda_{n-2 k}^{(k)} Q$, we have

$$
\begin{aligned}
L_{-k}\left[\left(1-\|x\|^{2}\right)^{k} Q(x)\right] & =\left(1-\|x\|^{2}\right)^{k}\left[-2 k d Q(x)+L_{k} Q(x)\right] \\
& =\left(1-\|x\|^{2}\right)^{k}[-2 k d-(n-2 k)(n-2 k+2 k+d)] Q(x) \\
& =-n(n-2 k+d)\left(1-\|x\|^{2}\right)^{k} Q(x),
\end{aligned}
$$

so that elements in $\left(1-\|x\|^{2}\right)^{k} \mathcal{V}_{n-2 k}^{d}\left(W_{k}\right)$ satisfy $L_{-k} P=\lambda_{n}^{(-k)} P$. Furthermore, by Lemma 3.2, elements of $\mathcal{H}_{n}^{d}$ are also eigenfunctions of $L_{-k}$ with eigenvalue $\lambda_{n}^{(-k)}$. Now let

$$
Z_{j}(x)=\left(1-\|x\|^{2}\right)^{j} Y(x)+\sum_{\nu=0}^{j-1} a_{j, \nu}^{n}\left(1-\|x\|^{2}\right)^{\nu} Y(x), \quad Y \in \mathcal{H}_{n-2 j}^{d} .
$$

We want to show that $Z_{j}$ satisfies the same equation. Applying (3.3) shows that

$$
\begin{aligned}
L_{-k} Z_{j}(x)= & -n(n-2 k+d)\left(1-\|x\|^{2}\right)^{j} Y(x)+\sum_{\nu=0}^{j}\left[4(\nu+1)(-k+\nu+1) a_{j, \nu+1}^{n}\right. \\
& \left.-(n-2 j+2 \nu)(n-2 j+2 \nu-2 k+2) a_{j, \nu}^{n}\right]\left(1-\|x\|^{2}\right)^{\nu} Y(x),
\end{aligned}
$$

so that the equation $L_{-k} Z_{j}=\lambda_{n}^{(-k)} Z_{j}$ implies, upon comparing the coefficients, that the $a_{j, \nu}^{(n)}$ satisfy the recursive relation

$$
a_{j, \nu}^{(n)}=a_{j, \nu+1}^{(n)} \frac{-2(\nu+1)(-k+\nu+1)}{(j-\nu)(2 n-2 j+2 \nu-2 k+d)} .
$$

Setting $a_{j, j}^{(n)}=1$, we can then determine $a_{j, \nu}^{(n)}$ recursively. The result is (3.2). This shows that all elements in $\mathcal{U}_{n}^{d}\left(W_{-k}\right)$ satisfy the equation $L_{-k} P=\lambda_{n}^{(-k)} P$.

If $a_{j, \nu}^{n}$ are all finite, then it follows from $\operatorname{dim} \mathcal{P}_{n}^{d}=\operatorname{dim} \mathcal{H}_{n}^{d}+\operatorname{dim} \mathcal{P}_{n-2}^{d}$ that

$$
\operatorname{dim} \mathcal{U}_{n}^{d}\left(W_{-k}\right)=\operatorname{dim} \mathcal{H}_{n}^{d}+\cdots+\operatorname{dim} \mathcal{H}_{n-2 k+2}^{d}+\operatorname{dim} \mathcal{P}_{n-2 k}^{d}=\operatorname{dim} \mathcal{P}_{n}^{d} .
$$

The explicit formula (3.2) shows that $a_{j, \nu}^{n}$ is not finite only if $n-j-k+\nu+d / 2$ is zero, which can happen only if $d$ is even and $n<j+k-\nu-d / 2$. Since $0 \leq \nu \leq j-1$ and $j \leq k-1$, this could happen only if $n-2 k-1+d / 2$ is zero or a negative integer. 
Some remarks are in order. If $d$ is even, then, as shown in the proof, $\mathcal{U}_{n}^{d}\left(W_{-k}\right)$ is not well defined if $n-j-k+d / 2=0$. The first time this happens is when $d=2$ and $k=2$. As $1 \leq j \leq k-1$, this happens when $j=1$ and $n=2$. In fact, for $k=2$, we have

$$
\mathcal{U}_{n}^{2}\left(W_{-2}\right)=\mathcal{H}_{n}^{2} \cup\left[\left(1-\|x\|^{2}\right)+\frac{1}{n-2}\right] \mathcal{H}_{n-2}^{2} \cup\left(1-\|\left. x\right|^{2}\right)^{2} \mathcal{V}_{n-4}^{2}\left(W_{2}\right),
$$

so that $\mathcal{U}_{2}^{d}\left(W_{-2}\right)$ is not well defined. Furthermore, since $\lambda_{2}^{(-2)}=0, \mathcal{H}_{2}=\operatorname{span}\left\{x_{1}^{2}-\right.$ $\left.x_{2}^{2}, 2 x_{1} x_{2}\right\}$ and $L_{\mu} 1=0$, we see that the linearly independent eigenfunctions with respect to the eigenvalue $\lambda_{2}^{(-2)}=0$ are $1, x_{1}^{2}-x_{2}^{2}, 2 x_{1} x_{2}$. However, since 1 is also the eigenfunction of $\lambda_{0}^{(-k)}$, we see that $L_{-2}$ does not have a complete polynomial basis as eigenfunctions and it misses exactly one polynomial of degree 2 .

The same analysis also works for $k \geq 2$. For example, for $k=4$ and $d=2$,

$$
\begin{aligned}
\mathcal{U}_{n}^{2}\left(W_{-4}\right)=\mathcal{H}_{n}^{2} \cup & {\left[\left(1-\|x\|^{2}\right)+\frac{3}{n-4}\right] \mathcal{H}_{n-2}^{2} } \\
\cup & {\left[\left(1-\|\left. x\right|^{2}\right)^{2}+\frac{4}{n-4}\left(1-\|x\|^{2}\right)+\frac{8}{(n-4)(n-5)}\right] \mathcal{H}_{n-4}^{2} } \\
\cup & {\left[\left(1-\|\left. x\right|^{2}\right)^{3}+\frac{3}{n-4}\left(1-\|x\|^{2}\right)^{2}+\frac{6}{(n-4)(n-5)}\left(1-\|x\|^{2}\right)\right.} \\
& \left.+\frac{6}{(n-4)(n-5)(n-6)}\right] \mathcal{H}_{n-6}^{2} \cup\left(1-\|\left. x\right|^{2}\right)^{2} \mathcal{V}_{n-8}^{d}\left(W_{2}\right),
\end{aligned}
$$

which shows clearly that the operator $L_{-4}$ has a complete basis of polynomials of degree exactly $n$ as eigenfunctions with respect to the eigenvalue $\lambda_{n}^{(-k)}$ only if $n>6$.

In general, for even dimension $d$, the operator $L_{-} k$ has a complete basis of polynomials of degree exactly $n$ as eigenfunctions only if $n>2 k-1+d / 2$. As a consequence, the eigenfunctions of the operator $L_{-k}$ do not contain a complete basis of polynomials if $d$ is even.

\section{Orthogonal POLYNOMials ASSOCIATED With LAPLACIAN}

For all $\mu \in \mathbb{R}$, the function $W_{\mu}$ is a symmetric factor for the differential operator $L_{\mu}$ as it is easy to verify that $L_{\mu} W_{\mu}=\left(W_{\mu} L_{\mu}\right)^{*}$ with

$$
\left(W_{\mu} L_{\mu}\right)^{*}:=\Delta W_{\mu}+(d+2 \mu)\langle\nabla, x\rangle W_{\mu}-\langle\nabla, x\rangle^{2} W_{\mu} .
$$

The verification is formal and holds for all $\mu \in \mathbb{R}$. The polynomial eigenfunctions of $L_{\mu}$ are orthogonal with respect to $W_{\mu}$ for $\mu>-1$. According to Theorem 3.3 , the polynomial eigenfunctions of $L_{-1}$ form a complete orthogonal basis with respect to $\langle\cdot, \cdot\rangle_{-1}$. It is natural to ask if the same holds true for $L_{-k}$ with $k=2,3, \ldots$ for $d$ being odd. Below we consider the case $k=2$.

Recall that in the case of $k=2$, the eigenfunctions of $L_{-2}$ are given by

$$
\mathcal{U}_{n}^{d}\left(W_{-2}\right)=\mathcal{H}_{n}^{d} \cup\left[\left(1-\|x\|^{2}\right)+\frac{1}{n-3+\frac{d}{2}}\right] \mathcal{H}_{n-2}^{d} \cup\left(1-\|x\|^{2}\right)^{2} \mathcal{V}_{n-4}^{d}\left(W_{2}\right)
$$

We first consider the following closely related class of functions:

$$
\mathcal{V}_{n}^{d}\left(W_{-2}\right):=\mathcal{H}_{n}^{d} \cup\left(1-\|x\|^{2}\right) \mathcal{H}_{n-2}^{d} \cup\left(1-\|x\|^{2}\right)^{2} \mathcal{V}_{n-4}^{d}\left(W_{2}\right)
$$


The difference between $\mathcal{U}_{n}^{d}\left(W_{-2}\right)$ and $\mathcal{V}_{n}^{d}\left(W_{-2}\right)$ is only in the middle term. Notice that the $\mathcal{V}_{n}^{d}\left(W_{-2}\right)$ are defined for all $n$ and $d$. It turns out that it is the space of orthogonal polynomials with respect to the inner product

$$
\langle f, g\rangle_{-2}:=\frac{\lambda}{\omega_{d}} \int_{B^{d}} \Delta f(x) \Delta g(x) d x+\frac{1}{\omega_{d}} \int_{S^{d-1}} f(x) g(x) d \omega, \quad \lambda>0,
$$

which is normalized so that $\langle 1,1\rangle_{-2}=1$. It is easy to see that this inner product is well-defined and is positive definite.

Theorem 4.1. The elements of $\mathcal{V}_{n}^{d}\left(W_{-2}\right)$ are orthogonal polynomials with respect to $\langle\cdot, \cdot\rangle_{-2}$. Moreover, they contain an orthonormal basis; in other words,

$$
\mathcal{V}_{n}^{d}\left(W_{-2}\right)=\mathcal{H}_{n}^{d} \oplus\left(1-\|x\|^{2}\right) \mathcal{H}_{n-2}^{d} \oplus\left(1-\|x\|^{2}\right)^{2} \mathcal{V}_{n-4}^{d}\left(W_{2}\right)
$$

Proof. First we make an observation that, by (2.5),

$$
\mathcal{V}_{n}^{d}\left(W_{-2}\right)=\mathcal{H}_{n}^{d} \cup\left(1-\|x\|^{2}\right) \mathcal{V}_{n-2}^{d}(\Delta) .
$$

We then prove that each of the two components on the right hand side of the above expression are orthogonal to lower degree polynomials and they are mutually orthogonal with respect to $\langle\cdot, \cdot\rangle_{-2}$. We consider the two cases separately.

Case 1. Let $P=Y_{n} \in \mathcal{H}_{n}^{d}$. Since $\Delta P=0,\langle P, g\rangle_{-2}=\frac{1}{\omega_{d}} \int_{S^{d-1}} f(x) g(x) d \omega$. If $g \in \Pi_{n-1}^{d}$, then $\langle P, g\rangle_{-2}=0$ by the orthogonality of $Y_{n}$. If $g \in\left(1-\|x\|^{2}\right) \mathcal{V}_{n-2}^{d}(\Delta)$, then the restriction of $g$ on $S^{d-1}$ is zero, so that $\langle P, g\rangle_{-2}=0$.

Case 2. Let $P(x)=\left(1-\|x\|^{2}\right) Q_{n-2}(x)$, where $Q_{n-2} \in \mathcal{V}_{n-2}^{d}(\Delta)$. Then the restriction of $P$ on $S^{d-1}$ is zero, so that

$$
\langle P, g\rangle_{-2}=\frac{\lambda}{\omega} \int_{B^{d}} \Delta\left[\left(1-\|x\|^{2}\right) Q_{n-2}(x)\right] \Delta g(x) d x .
$$

Let $g \in \mathcal{V}_{m}^{d}\left(W_{-2}\right)$ and $m<n$. If $g \in \mathcal{H}_{m}$, then $\Delta g=0$ so that $\langle P, g\rangle_{-2}=0$. If $g \in\left(1-\|x\|^{2}\right) \mathcal{V}_{m-2}^{d}(\Delta)$, then $g(x)=\left(1-\|x\|^{2}\right) h(x)$ for $h \in \mathcal{V}_{m-2}^{d}(\Delta)$. Recalling the definition of $\langle f, g\rangle_{\Delta}$ in (2.4), we then have $\langle P, g\rangle_{-2}=\left\langle Q_{n-2}, h\right\rangle_{\Delta}=0$ by the orthogonality of $Q_{n-2} \in \mathcal{V}_{n-2}^{d}(\Delta)$.

Recall that $\mathcal{V}_{n}^{d}\left(W_{-2}\right)$ and $\mathcal{U}_{n}^{d}\left(W_{-2}\right)$ differ only at the middle component. We do not know if there is an inner product with respect to which $\mathcal{U}_{n}^{d}\left(W_{-2}\right)$ is orthogonal for all $n$. For each fixed $n \in \mathbb{N}$, however, elements of $\mathcal{U}_{n}^{d}\left(W_{-2}\right)$ are orthogonal to lower degree polynomials with respect to the following inner product:

$$
\begin{aligned}
\langle f, g\rangle_{n}^{*}:= & \frac{\lambda}{\omega_{d}} \int_{B^{d}} \Delta f(x) \Delta g(x) d x+\frac{1}{\omega_{d}} \int_{S^{d-1}} f(x) g(x) d \omega \\
& +\frac{\mu}{\omega_{d}} \int_{S^{d-1}} \frac{d}{d r}\left[r^{n-4-d} f(x)\right] \frac{d}{d r}\left[r^{n-4-d} g(x)\right] d \omega,
\end{aligned}
$$

where $\frac{d}{d r}$ is the normal derivative, $\lambda>0$ and $\mu>0$. It is easy to see that $\langle f, g\rangle_{n}^{*}$ is positive definite for $f, g \in \Pi_{n}^{d}$.

Theorem 4.2. The elements of $\mathcal{V}_{n}^{d}\left(W_{-2}\right)$ are orthogonal polynomials with respect to the inner product $\langle f, g\rangle_{n}^{*}$. 
Proof. Notice that the first two terms in the $\langle f, g\rangle_{n}^{*}$ are exactly $\langle f, g\rangle_{-2}$. For $x \in \mathbb{R}^{d}$, write $x=r x^{\prime}, r>0$ and $x^{\prime} \in S^{d-1}$. We assume that $g \in \Pi_{n-1}^{d}$ below. If $P=Y_{n} \in$ $\mathcal{H}_{n}^{d}$, then $\frac{d}{d r}\left[r^{n-4-d} P(x)\right]=(2 n-4-d) Y_{n}\left(x^{\prime}\right)$ on $S^{d-1}$. Hence $\langle P, g\rangle_{n}^{*}=0$ as $\Delta P=$ 0 and $P \in C H_{n}^{d}$. If $P \in\left(1-\|x\|^{2}\right)^{2} \mathcal{V}_{n-4}^{d}\left(W_{2}\right)$, then $\frac{d}{d r}\left[r^{n-4-d} P(x)\right]=0$ on $S^{d-1}$ because of the $\left(1-r^{2}\right)^{2}$ term, so that $\langle P, g\rangle_{n}^{*}=\langle P, g\rangle_{-2}=0$. Thus, by (4.1), we are left with the case $P(x)=\left[\left(1-\|x\|^{2}\right)+\frac{1}{n-3+d / 2}\right] Y_{n-2}(x)$, where $Y_{n-2} \in \mathcal{H}_{n-2}^{d}$. Since $Y_{n-2}$ is homogeneous, $Y_{n-2}(x)=r^{n-2} Y_{n-2}\left(x^{\prime}\right)$, a simple computation shows that $\frac{d}{d r}\left[r^{n-4-d} P(x)\right]=0$. Consequently, we again have $\langle P, g\rangle_{n}^{*}=\langle P, g\rangle_{-2}=0$.

These results indicate that there is a difference between the case $k=-1$ and $k=$ $-2,-3, \ldots$. Not only does the differential operator $L_{-k}$ have complete polynomial eigenfunctions only when $d$ is odd in the latter case, the eigenfunctions also may no longer be orthogonal with respect to an inner product for all $n$.

Another interesting question is whether or not the main terms of $\mathcal{U}_{n}^{d}\left(W_{-k}\right)$ are orthogonal for $k \geq 3$. In other words, we ask if the spaces

$$
\mathcal{V}_{n}^{d}\left(W_{-k}\right):=\mathcal{H}_{n}^{d} \cup\left(\bigcup_{j=1}^{k-1}\left(1-\|x\|^{2}\right)^{j} \mathcal{H}_{n-2 j}^{d}\right) \cup\left(1-\|x\|^{2}\right)^{k} \mathcal{V}_{n-2 k}^{d}\left(W_{k}\right)
$$

are orthogonal with respect to a positive definite inner product, which will likely involve higher order derivatives, as indicated by our result for $k=1$ and $k=2$. At this point we do not see a way to find such an inner product.

\section{ACKNOWLEDGMENT}

The authors thank Jeong Keun Lee for communicating and discussing the results in 9 .

\section{REFERENCES}

[1] M. Alfaro, M. Álvarez de Morales and M. L. Rezola, Orthogonality of the Jacobi polynomials with negative integer parameters. J. Comput. Appl. Math. 145 (2002), 379-386. MR1917278 (2003h:33006)

[2] M. Alfaro, T. E. Pérez, M. A. Piñar and M. L. Rezola, Sobolev orthogonal polynomials: The discrete-continuous case, Methods Appl. Anal. 6 (1999), 593-616. MR.1795525(2001i:42035)

[3] M. Álvarez de Morales, T. E. Pérez and M. A. Piñar, Sobolev orthogonality for the Gegenbauer polynomials $\left\{C_{n}^{(-N+1 / 2)}\right\}_{n \geq 0}$, J. Comput. Appl. Math. 100 (1998), 111-120. MR.1658734 (99k:33019)

[4] K. Atkinson and O. Hansen, Solving the nonlinear Poisson equation on the unit disk, J. Integral Equations Appl. 17 (2005), 223-241. MR2213625 (2006m:35082)

[5] C. F. Dunkl and Yuan Xu, Orthogonal polynomials of several variables, Encyclopedia of Mathematics and its Applications, vol. 81, Cambridge Univ. Press, 2001. MR 1827871 (2002m:33001)

[6] I. H. Jung, K. H. Kwon, and J. K. Lee, Sobolev orthogonal polynomials relative to $\lambda p(c) q(c)+$ $\left\langle\tau, p^{\prime}(x) q^{\prime}(x)\right\rangle$, Comm. Korean Math. Soc. 12 (1997), 603-617. MR1641942 (99f:42050)

[7] H. L. Krall and I. M. Sheffer, Orthogonal polynomials in two variables. Ann. Mat. Pura Appl. (4) 76 (1967), 325-376. MR0228920 (37:4499)

[8] J. K. Lee and L. L. Littlejohn, Sobolev orthogonal polynomials in two variables and second order partial differential equations, J. Math. Anal. Appl. 322 (2006), 1001-1017. MR2250632 (2007e:42028)

[9] J. K. Lee, L. L. Littlejohn, J. H. Park, H. T. Shim and B. H. Yoo, Real weight functions for the circle polynomials by the regularization, to appear.

[10] G. Szegő, Orthogonal Polynomials, 4th edition, Amer. Math. Soc. Colloq. Publ., Vol. 23, Amer. Math. Soc., Providence, RI, 1975. MR0372517 (51:8724) 
[11] Yuan Xu, A family of Sobolev orthogonal polynomials on the unit ball, J. Approx. Theory 138 (2006), 232-241. MR 2201161 (2006k:42015)

[12] Yuan Xu, Sobolev orthogonal polynomials defined via gradient on the unit ball, J. Approx. Theory 152 (2008), 52-65. MR2419297

Department of Applied Mathematics, University of Granada, Granada 18071, Spain

E-mail address: mpinar@ugr.es

Department of Mathematics, University of Oregon, Eugene, Oregon 97403-1222

E-mail address: yuan@math.uoregon.edu 